\title{
Talk about Several Time Periodic Pulse Electroosmotic Flow of Maxwell Fluid in a Circular Microchannel
}

\author{
Dongsheng Li, Kun Li ${ }^{*}$ \\ College of Science, Inner Mongolia University of Technology, Hohhot, China \\ Email: ^jadewh@163.com
}

How to cite this paper: Li, D.S. and Li, K. (2021) Talk about Several Time Periodic Pulse Electroosmotic Flow of Maxwell Fluid in a Circular Microchannel. Journal of Applied Mathematics and Physics, 9, 617-634. https://doi.org/10.4236/jamp.2021.94045

Received: March 19, 2021

Accepted: April 17, 2021

Published: April 20, 2021

Copyright (c) 2021 by author(s) and Scientific Research Publishing Inc. This work is licensed under the Creative Commons Attribution International License (CC BY 4.0).

http://creativecommons.org/licenses/by/4.0/

\section{(c) (i) Open Access}

\begin{abstract}
Using the method of Laplace transform, analytical expressions are derived for the time periodic pulse electroosmotic flow (EOF) velocity of the triangle and sawtooth of Maxwell fluid in circular microchannel. The solution involves analytically solving the linearized Poisson-Boltzmann (P-B) equation, together with the Cauchy momentum equation and the general Maxwell constitutive equation. By numerical computations of inverse Laplace transform, the effects of electrokinetic width $K$, relaxation time $\bar{\lambda}_{1}$ and pulse width $a$ on the above several pulse EOF velocities are investigated. In addition, we focused on the comparison and analysis of the formulas and graphs between the triangle and sawtooth pulse EOF with the rectangle pulse EOF. The study found that there are obvious differences in formulas and graphs between triangle and sawtooth pulse EOF with rectangle pulse EOF, and the difference mainly depends on the different definitions of the three kinds of time periodic pulse waves. Finally, we also studied the stability of the above three kinds of pulse EOF and the influence of relaxation time on pulse EOF velocity under different pulse widths is discussed. We find that the rectangle pulse EOF is more stable than the triangle and sawtooth pulse EOF. For any pulse, as the pulse width $a$ increases, the influence of the relaxation time on the pulse EOF velocity will be weakened.
\end{abstract}

\section{Keywords}

Pulse Electroosmotic Flow, Maxwell Fluid, Circular Microchannel, Relaxation Time, Pulse Width

\section{Introduction}

Microfluidic devices have become increasingly more important because of their 
application in micro-electro-mechanical systems (MEMS) and microbiological sensors (such as lab-on-a-chip) [1] [2]. In general, when most substances come into contact with polar solutions, they tend to generate negative charges on the surface. The distribution of ions near the wall in the solution will be affected by this phenomenon. The opposite ions with the opposite polarity to the wall will be attracted to the wall, while the same ions will be repelled away from the wall. In this way, an electric double layer (EDL) will be formed [3]. Further, when an external electric field is applied to both ends of the channel, the ions in the electric double layer will move under the force of the electric field. This is due to the viscosity of the fluid itself, the moving free ions will drive the movement of nearby fluid clusters, and eventually form an electro-osmotic flow (EOF).

In previous research, a large number of theoretical and experimental studies [4]-[9] on the fully developed EOF problem of Newtonian fluids in various geometric shapes of microchannels have been completed. However, this steady EOF problem requires higher voltage and larger field strength, which may bring many difficulties to the experimental conditions. Very recently, time-dependent EOF as an alternative mechanism of microfluidic transport has attracted the attention of scholars at home and abroad [10]-[15].

All of the above-mentioned studies are related to Newtonian fluids. But most of solutions of industry and biopharmaceutical are fluid that has the structural characteristic of non-Newtonian fluids, such as biological fluid and other solutions of long-chain molecules, which structural characteristics include strain force, normal shear stress, hysteresis effect, variable viscosity, memory effect and so on [16]. Therefore, the study of non-Newtonian fluids becomes very important. Additionally, the theoretical research of electroosmotic flow of non-Newtonian fluids is mainly limited to simple fluid models because of the inherent analytical difficulties introduced by more complex constitutive equation [17]. So far, some work has been done on the simple fluid models, for example Oldroyd-B fluid model [18] [19] [20], Power-law fluid model [21] [22] [23], Maxwell fluid model [24] [25] [26] [27] and Jeffrey fluid model [28] [29] [30].

Although some basic characteristics of EOF of non-Newtonian fluids have been reported in the above studies, its rich properties still need to be examined. Recent study have shown that Maxwell fluid model simulation of blood in narrow conical vessels has achieved an ideal effect, and it is completely possible to analyze the blood-based microfluidics and other microbial fluid transmission systems by means of electric mechanism [30]. At the same time, the literature [31] shows that low-frequency pulses can promote local blood circulation, which has been proved in clinical medical research. Thus, the study of Maxwell fluid pulse EOF will play a very beneficial role in blood transport.

However, from the current research situation, there is almost no research on pulse EOF, and it has not attracted enough attention from the majority of researchers. Therefore, based on the rectangle pulse EOF, we have re-selected several common pulses (such as triangle pulse and sawtooth pulse) to study the time periodic pulse EOF of Maxwell fluid through a circular microchannel in 
present work. The target of this article is to derive the semi-analytical solutions of the above two time periodic pulse EOF for viscoelastic fluid. Meanwhile, we analyzed the effect of several parameters such as the electrokinetic width, the relaxation time and the pulse width on the pulse EOF of Maxwell fluid. Moreover, we also discussed the different effects of relaxation time on the EOF velocity of different pulses. It is mainly compared with the rectangle pulse EOF [32] completed before, and some new results have been found. In the second section, the physical model of the problem is described and the semi-analytical solutions of the governing equations of rectangle pulse EOF, triangle pulse EOF and sawtooth pulse EOF are derived. In the third section, the article discusses numerical results and the parametric which dependent on the pulse EOF velocity for different pulses. At last, in the fourth section, the article presents the conclusions.

\section{Problem Formulation}

\subsection{Cauchy Momentum Equation and Constitutive Relation}

The time periodic pulse EOF of an incompressible Maxwell fluid through a circular microchannel is sketched in Figure 1. The channel has a circular cross-section with a radius $R$ and a length $L$, assumed to be much larger than the diameter i.e., $L \gg 2 R$. As shown in Figure 2, the pulse EOF is pumped through several pulse electric fields of strength $E_{0}$ with pulse amplitude of 1 , pulse repetition period of $2 a$ and pulse width of $a$, respectively.

Due to the symmetry of the geometry, we only study the semi-section of the microchannel. Provided that the pressure gradient along $z$ direction is ignored, then the one-dimensional Cauchy momentum equation can be written as

$$
\rho \frac{\partial u(r, t)}{\partial t}=-\frac{1}{r} \frac{\partial}{\partial r}\left(r \tau_{r z}\right)+\rho_{e}(r) E_{0} f(t)
$$

where $u(r, t)$ is the velocity along $z$ axial direction, $\rho$ is the fluid density, $t$ is the time, $\tau_{r z}$ is the stress tensor and $\rho_{e}(r)$ is the volume charge density, $E_{0} f(t)$ is the ideal pulsed electric field of strength $E_{0}$.

Provided that the boundary condition of Equation (1) is no slip, and it can be given as [33]

$$
\left.u(r, t)\right|_{r=R}=0,\left.\frac{\partial u(r, t)}{\partial r}\right|_{r=0}=0
$$

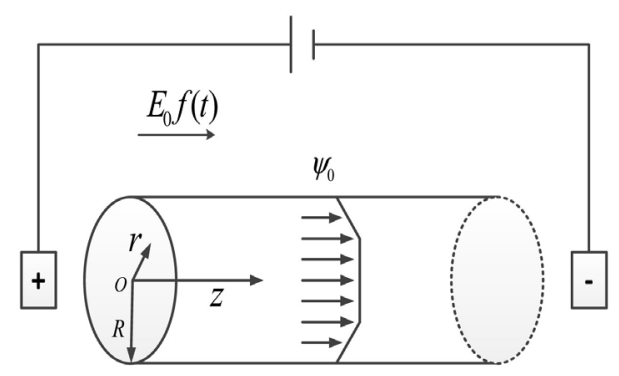

Figure 1. Schematic of the time periodic pulse EOF of Maxwell fluid in a circular microchannel. 


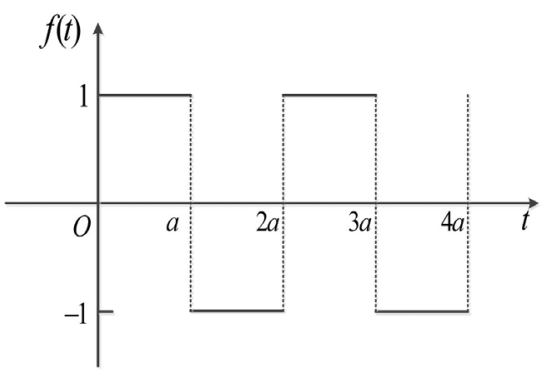

(a)

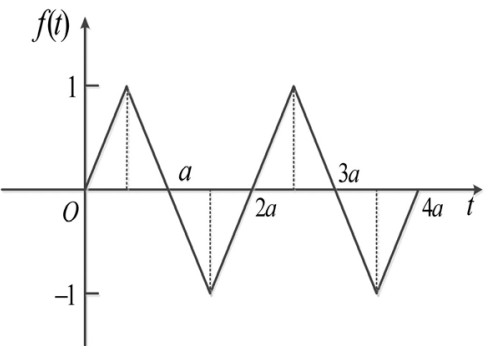

(b)

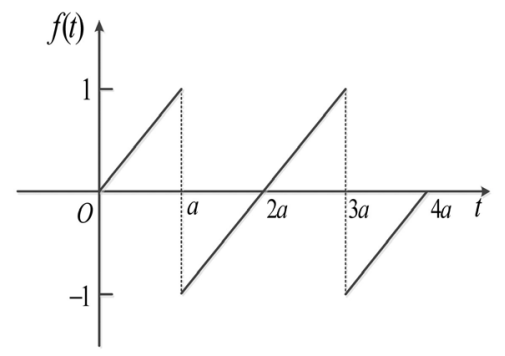

(c)

Figure 2. The diagram of three kinds of time periodic pulse waves. (a) Ideal rectangle pulse wave; (b) Ideal triangle pulse wave; (c) Ideal sawtooth pulse wave.

For the Maxwell fluid, the constitutive equation satisfies [34]

$$
\tau_{r \mathrm{Z}}+\lambda_{1} \frac{\partial}{\partial t} \tau_{r \mathrm{z}}=-\eta_{0} \frac{\partial u(r, t)}{\partial r}
$$

where $\lambda_{1}$ is the relaxation time, $\eta_{0}$ is zero shear rate viscosity.

\subsection{Electric Potential Field Solution}

The chemical interaction between the electrolyte liquid and the solid wall produces an electric double layer (EDL), a very thin layer of charged liquid at the solid-liquid interface. A cylindrical coordinate system $(r, \theta, z)$ is adopted. In this theoretical model, it is assumed that the channel wall is uniformly charged, so that the electrical potential in the EDL only varies in this $r$ direction and does not depend on $\theta$ [33]. For a symmetric binary electrolyte solution, assuming that the electrical potential $\psi$ of the EDL is stable, and its distribution and the local volumetric net charge density $\rho_{e}(r)$ are described by the PoissonBoltzmann (P-B) equation

$$
\begin{gathered}
\frac{1}{r} \frac{\mathrm{d}}{\mathrm{d} r}\left(r \frac{\mathrm{d} \psi(r)}{\mathrm{d} r}\right)=-\frac{\rho_{e}(r)}{\varepsilon} \\
\rho_{e}(r)=-2 n_{0} z_{v} e_{0} \sinh \left[\frac{z_{v} e_{0} \psi(r)}{k_{b} T}\right]
\end{gathered}
$$

where $\varepsilon$ is the dielectric constant of the electrolyte liquid, $\psi(r)$ is the electrical potential of the EDL, $n_{0}$ is the ion density of the bulk liquid, $z_{v}$ is the valence, $e_{0}$ is the electron charge, $k_{b}$ is the Boltzmann constant, $T$ is the absolute temperature, and sinh is the sine function. 
Combing Equation (4) and Equation (5) gives

$$
\frac{1}{r} \frac{\mathrm{d}}{\mathrm{d} r}\left(r \frac{\mathrm{d} \psi(r)}{\mathrm{d} r}\right)=\frac{2 n_{0} z_{v} e_{0}}{\varepsilon} \sinh \left[\frac{z_{v} e_{0} \psi(r)}{k_{b} T}\right]
$$

which is subject to the following boundary conditions

$$
\left.\psi(r)\right|_{r=R}=\psi_{0},\left.\frac{\mathrm{d} \psi(r)}{\mathrm{d} r}\right|_{r=0}=0
$$

where $\psi_{0}$ is wall zeta potential, $r$ is radial coordinate and $R$ is radius of the circular microchannel.

Assuming that the electrical potential is small enough, the Debye-Hückel linearization approximation can be applied to the hyperbolic sine function appearing on the right hand side of Equation (6), which means that the electrical potential is physically small compared to the thermal energy of the charged species [28]. Thus, Equation (6) can be simplified as

$$
\frac{1}{r} \frac{\mathrm{d}}{\mathrm{d} r}\left(r \frac{\mathrm{d} \psi(r)}{\mathrm{d} r}\right)=\kappa^{2} \psi(r), \text { and } \kappa=\left(\frac{2 n_{0} z_{v}^{2} e_{0}^{2}}{\varepsilon k_{b} T}\right)^{1 / 2}
$$

where $\kappa$ is the Debye-Hückel parameter, which $1 / \kappa$ usually represents the thickness of the EDL in physical.

By solving Equation (7) and Equation (8), the net charge density distribution for circular microchannel can be express as

$$
\rho_{e}(r)=-\varepsilon \kappa^{2} \psi_{0} \frac{I_{0}(\kappa r)}{I_{0}(\kappa R)}
$$

where $I_{0}$ is the first kind modified Bessel function of order zero.

\subsection{The Analytical Solutions of the Cauchy Momentum Equation}

In order to obtain the solution of the velocity field of the triangle pulse EOF and the sawtooth pulse EOF, let us first briefly review the process of solving the velocity field of the rectangle pulse EOF, and then analyze the difference among the three formulas to obtain the corresponding velocity field solution above.

\subsubsection{Rectangle Pulse Wave}

The ideal rectangle pulse can be expressed as the following form

$$
f(t)= \begin{cases}1, & t \in[0, a) \\ -1, & t \in(a, 2 a] .\end{cases}
$$

For simplicity, the following dimensionless groups are introduced:

$$
\begin{aligned}
& \bar{r}=\frac{r}{R}, K=\kappa R,\left(\bar{t}, \bar{\lambda}_{1}\right)=\frac{\left(t, \lambda_{1}\right)}{\rho R^{2} / \eta_{0}}, \bar{u}(\bar{r}, \bar{t})=\frac{u(r, t)}{U_{e o}}, \\
& \bar{\tau}_{r z}=\frac{\tau_{r z}}{\eta_{0} U_{e o} / R}, U_{e o}=-\frac{\varepsilon \psi_{0} E_{0}}{\eta_{0}}
\end{aligned}
$$

where $U_{e o}$ denotes steady Helmholtz-Smoluchowshi EOF velocity of Newtonian fluids, $K$ is the ratio of the characteristic width of the microchannel to 
Debye length.

Using Equation (11), Equations of (1) and (3) and boundary conditions (2) are normalized as

$$
\begin{gathered}
\frac{\partial \bar{u}(\bar{r}, \bar{t})}{\partial \bar{t}}=-\frac{1}{\bar{r}} \frac{\partial}{\partial \bar{r}}\left(\bar{r} \overline{\tau_{\overline{r z}}}\right)+f(\bar{t}) K^{2} \frac{I_{0}(K \bar{r})}{I_{0}(K)} \\
\overline{\tau_{\overline{r z}}}+\overline{\lambda_{1}} \frac{\partial}{\partial \bar{t}} \overline{\tau_{\overline{r z}}}=-\frac{\partial \bar{u}(\bar{r}, \bar{t})}{\partial \bar{r}} \\
\left.\bar{u}(\bar{r}, \bar{t})\right|_{\bar{r}=1}=0,\left.\frac{\partial \bar{u}(\bar{r}, \bar{t})}{\partial \bar{r}}\right|_{\bar{r}=0}=0
\end{gathered}
$$

Eliminating $\overline{\tau_{\overline{r z}}}$ from Equation (12) and Equation (13) yields

$$
\begin{aligned}
& \frac{\partial \bar{u}(\bar{r}, \bar{t})}{\partial \bar{t}}+\bar{\lambda}_{1} \frac{\partial^{2} \bar{u}(\bar{r}, \bar{t})}{\partial \bar{t}^{2}} \\
& =\frac{1}{\bar{r}} \frac{\partial}{\partial \bar{r}}\left(\bar{r} \frac{\partial \bar{u}(\bar{r}, \bar{t})}{\partial \bar{r}}\right)+\left(1+\bar{\lambda}_{1} \frac{\partial}{\partial \bar{t}}\right) f(\bar{t}) K^{2} \frac{I_{0}(K \bar{r})}{I_{0}(K)}
\end{aligned}
$$

Let us employ the method of Laplace transforms defined by

$$
U(\bar{r}, s)=L[\bar{u}(\bar{r}, \bar{t})]=\int_{0}^{\infty} \bar{u}(\bar{r}, \bar{t}) \mathrm{e}^{-s \bar{t}} \mathrm{~d} \bar{t}
$$

Obviously $\frac{\partial}{\partial \bar{t}} f(\bar{t})=0$ in Equation (15), and the Laplace transform of $f(\bar{t})$ is given by the Appendix A.

From the literature [32], the solution of the velocity field is given as

$$
U(\bar{r}, s)=\frac{\tanh \left(\frac{a s}{2}\right) K^{2}}{s\left(K^{2}-\beta^{2}\right)}\left(\frac{I_{0}(\beta \bar{r})}{I_{0}(\beta)}-\frac{I_{0}(K \bar{r})}{I_{0}(K)}\right)
$$

where $\beta=\sqrt{\bar{\lambda}_{1} s^{2}+s}$, tanh is a hyperbolic tangent function.

The inverse Laplace transform is defined by

$$
\bar{u}(\bar{r}, \bar{t})=L^{-1}[U(\bar{r}, s)]=\frac{1}{2 \pi i} \int_{\Gamma} U(\bar{r}, s) \mathrm{e}^{s \bar{t}} \mathrm{~d} s
$$

where $\Gamma$ is a vertical line to the right of all singularities of $U(\bar{r}, s)$ in the complex $s$ plane. The exact solution of the EOF velocity cannot be obtained analytically due to the complexity of the express of $U(\bar{r}, s)$. Therefore, the numerical computation must be performed by numerical inverse Laplace transform [35].

\subsubsection{Triangle Pulse Wave}

The ideal triangle pulse can be expressed as the following form

$$
f(t)= \begin{cases}\frac{2}{a} t, & t \in\left[0, \frac{a}{2}\right), \\ 2-\frac{2}{a} t, & t \in\left[\frac{a}{2}, \frac{3 a}{2}\right], \\ \frac{2}{a} t-4, & t \in\left(\frac{3 a}{2}, 2 a\right] .\end{cases}
$$


The difference from the rectangle pulse wave is $\frac{\partial}{\partial \bar{t}} f(\bar{t}) \neq 0$ in Equation (15), the others are the same, and the Laplace transform of $\left(1+\frac{\partial \bar{t}}{\partial \bar{t}}\right) f(\bar{t})$ is given by the Appendix B.

If initial condition satisfies $\bar{u}(\bar{r}, 0)=0$, then the transforms of Equation (15) and Equation (14) can be written as

$$
\begin{gathered}
\bar{\lambda}_{1} s^{2} U(\bar{r}, s)+s U(\bar{r}, s) \\
=\frac{\partial^{2} U(\bar{r}, s)}{\partial \bar{r}^{2}}+\frac{1}{\bar{r}} \frac{\partial U(\bar{r}, s)}{\partial \bar{r}}+\frac{2\left(1+\overline{\lambda_{1}} s\right)\left(1-\operatorname{sech}\left(\frac{a s}{2}\right)\right) K^{2}}{a s^{2}} \frac{I_{0}(K \bar{r})}{I_{0}(K)} \\
\left.U(\bar{r}, s)\right|_{\bar{r}=1}=0,\left.\frac{\partial U(\bar{r}, s)}{\partial \bar{r}}\right|_{\bar{r}=0}=0
\end{gathered}
$$

Equation (20) can be simplified as

$$
\begin{aligned}
& \frac{\partial^{2} U(\bar{r}, s)}{\partial \bar{r}^{2}}+\frac{1}{\bar{r}} \frac{\partial U(\bar{r}, s)}{\partial \bar{r}}-\beta^{2} U(\bar{r}, s) \\
& =-\frac{2\left(1+\bar{\lambda}_{1} s\right)\left(1-\operatorname{sech}\left(\frac{a s}{2}\right)\right) K^{2}}{a s^{2}} \frac{I_{0}(K \bar{r})}{I_{0}(K)}
\end{aligned}
$$

where $\beta=\sqrt{\bar{\lambda}_{1} s^{2}+s}$, sech is a hyperbolic secant function.

Equation (22) is a linear and inhomogeneous ordinary differential equation, and its solution can be written as the sum of a general solution $U_{h}(\bar{r}, s)$ corresponding to homogeneous equation and a special solution $U_{s}(\bar{r}, s)$.

$$
U(\bar{r}, s)=U_{h}(\bar{r}, s)+U_{s}(\bar{r}, s)
$$

The homogeneous solution of Equation (22) is expressed as

$$
U_{h}(\bar{r}, \bar{s})=A I_{0}(\beta \bar{r})+B K_{0}(\beta \bar{r})
$$

where $I_{0}$ and $K_{0}$ are modified Bessel functions of first and second kinds of order zero, respectively.

Due to the finite of $U(\bar{r}, s)$ at $\bar{r}=0$, the constant $B$ equal to zero from the boundary condition Equation (21). Therefore, the homogeneous solution of Equation (22) is rewritten as

$$
U_{h}(\bar{r}, \bar{s})=A I_{0}(\beta \bar{r})
$$

here $A$ is constant, which can be determined from boundary conditions of Equation (21).

Considering the formation of the right hand side of Equation (22), the special solution can be given as

$$
U_{s}(\bar{r}, s)=C I_{0}(K \bar{r})
$$

Inserting Equation (26) into Equation (22) gives

$$
C\left[\frac{\mathrm{d}^{2} I_{0}(K \bar{r})}{\mathrm{d} \bar{r}^{2}}+\frac{1}{\bar{r}} \frac{\mathrm{d} I_{0}(K \bar{r})}{\mathrm{d} \bar{r}}-\beta^{2} I_{0}(K \bar{r})\right]=-\frac{2\left(1+\bar{\lambda}_{1} s\right)\left(1-\operatorname{sech}\left(\frac{a s}{2}\right)\right) K^{2}}{a s^{2}} \frac{I_{0}(K \bar{r})}{I_{0}(K)}
$$


From Equation (8) and Equation (11), we can get

$$
\frac{\mathrm{d}^{2} I_{0}(K \bar{r})}{\mathrm{d} \bar{r}^{2}}+\frac{1}{\bar{r}} \frac{\mathrm{d} I_{0}(K \bar{r})}{\mathrm{d} \bar{r}}=K^{2} I_{0}(K \bar{r})
$$

Substituting Equation (28) into Equation (27) and equalizing the coefficients in front of the modified Bessel functions $I_{0}(K \bar{r})$ at the two sides of the equation yields

$$
C=-\frac{2\left(1+\bar{\lambda}_{1} s\right)\left(1-\operatorname{sech}\left(\frac{a s}{2}\right)\right) K^{2}}{a s^{2}\left(K^{2}-\beta^{2}\right) I_{0}(K)}
$$

Thus, the solution of velocity $U(\bar{r}, \bar{s})$ can be written as

$$
U(\bar{r}, s)=A I_{0}(\beta \bar{r})-\frac{2\left(1+\bar{\lambda}_{1} s\right)\left(1-\operatorname{sech}\left(\frac{a s}{2}\right)\right) K^{2}}{a s^{2}\left(K^{2}-\beta^{2}\right) I_{0}(K)} I_{0}(K \bar{r})
$$

The coefficient $A$ with boundary condition of Equation (21) can be determined as

$$
A=\frac{2\left(1+\bar{\lambda}_{1} s\right)\left(1-\operatorname{sech}\left(\frac{a s}{2}\right)\right) K^{2}}{a s^{2}\left(K^{2}-\beta^{2}\right) I_{0}(\beta)}
$$

Inserting Equation (31) into Equation (30), we have

$$
U(\bar{r}, s)=\frac{2\left(1+\bar{\lambda}_{1} s\right)\left(1-\operatorname{sech}\left(\frac{a s}{2}\right)\right) K^{2}}{a s^{2}\left(K^{2}-\beta^{2}\right)}\left(\frac{I_{0}(\beta \bar{r})}{I_{0}(\beta)}-\frac{I_{0}(K \bar{r})}{I_{0}(K)}\right)
$$

where $\beta=\sqrt{\bar{\lambda}_{1} s^{2}+s}$, sech is a hyperbolic secant function.

As with rectangle pulse wave, the numerical computation must be performed by numerical inverse Laplace transform of Equation (32).

\subsubsection{Sawtooth Pulse Wave}

The ideal sawtooth pulse can be expressed as the following form

$$
f(t)= \begin{cases}\frac{t}{a}, & t \in[0, a), \\ \frac{t}{a}-2, & t \in(a, 2 a] .\end{cases}
$$

The Laplace transform of $\left(1+\frac{\partial}{\partial \bar{t}}\right) f(\bar{t})$ is given by the Appendix C.

Making the Laplace transform for Equation (15), we have

$$
\begin{aligned}
& \frac{\partial^{2} U(\bar{r}, s)}{\partial \bar{r}^{2}}+\frac{1}{\bar{r}} \frac{\partial U(\bar{r}, s)}{\partial \bar{r}}-\beta^{2} U(\bar{r}, s) \\
& =-\frac{\left(1+\bar{\lambda}_{1} s-a s \operatorname{csch}(a s)\right) K^{2}}{a s^{2}} \frac{I_{0}(K r)}{I_{0}(K)}
\end{aligned}
$$

where $\beta=\sqrt{\bar{\lambda}_{1} s^{2}+s}$, csch is a hyperbolic cosecant function. 
Therefore, the solution of Equation (34) with boundary condition (21) can be given as

$$
U(\bar{r}, s)=\frac{\left(1+\bar{\lambda}_{1} s-a s \operatorname{csch}(a s)\right) K^{2}}{a s^{2}\left(K^{2}-\beta^{2}\right)}\left(\frac{I_{0}(\beta \bar{r})}{I_{0}(\beta)}-\frac{I_{0}(K \bar{r})}{I_{0}(K)}\right)
$$

Similar to the above several pulse waves, the numerical calculation must also be performed by the numerical inverse Laplace transform of Equation (35).

\section{Results and Discussion}

In the previous section, we have obtained the semi-analytical solutions of the time periodic pulse EOF velocity of the triangle and sawtooth of Maxwell fluid through a circular microchannel, which rely mainly on electrokinetic width $K$, relaxation time $\bar{\lambda}_{1}$ and pulse width $a$. Then, we also compare with the rectangle pulse EOF on the graph, and discuss the influence of the above several parameters on the velocity distribution of different pulse EOF. Among them, it is important to analyze the influence of relaxation time on the three kinds of EOF under the condition of a fixed pulse width, that is, the stability. Additionally, the effect of relaxation time on pulse EOF under different pulse widths is discussed.

Figure 3 gives the variations of pulse EOF velocity with time for different pulses (rectangle pulse, triangle pulse and sawtooth pulse) when $K=20, a=1$

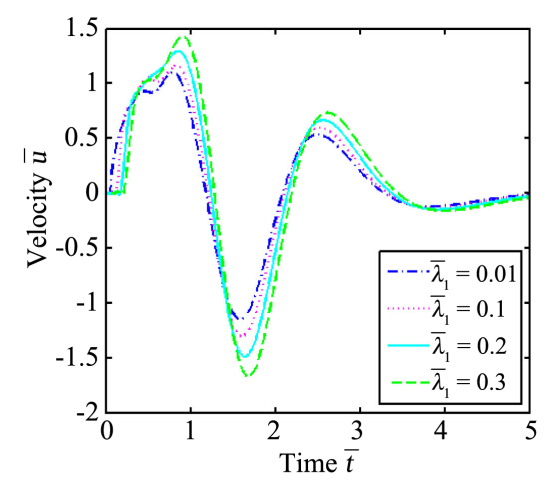

(a)

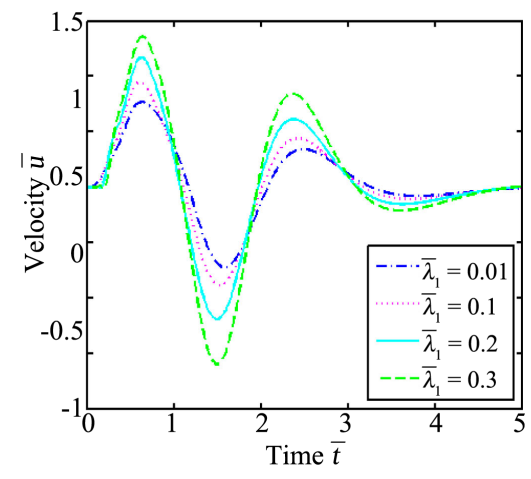

(b)

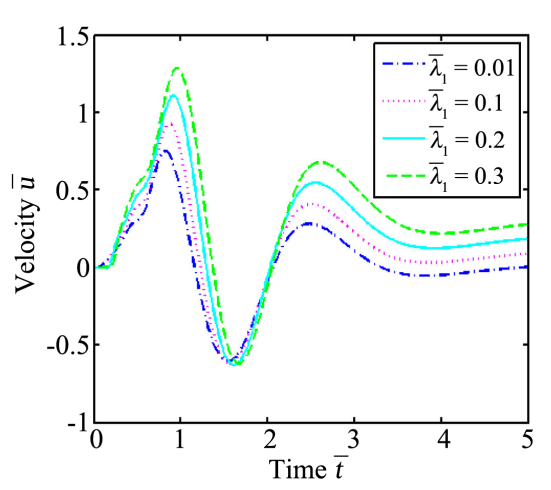

(c)

Figure 3. Variations of pulse EOF velocity $\bar{u}$ at different relaxation times with time for different pulses when $K=20, a=1$ and $\bar{r}=0.5$. (a) Rectangle pulse; (b) Triangle pulse; (c) Sawtooth pulse. 
and $\bar{r}=0.5$. We can clearly find that for different pulses, the variations of velocity are relatively significant. Therefore, it is very necessary to study different time periodic pulse EOF. At the same time, it can be seen from Figure 3 that for any pulse, the velocity amplitude increases with relaxation time $\bar{\lambda}_{1}$. The main reason is that the longer relaxation time, which means the greater elastic effect and weaker recovery ability of Maxwell fluid. Because of the "fading memory" phenomenon of Maxwell fluid, increasing the relaxation time leads more easily to the variation of the pulse EOF velocity profiles caused by external electric field [36]. Moreover, it is very obvious from Figure 3 that the velocity of three kinds of pulse EOF gradually attains to steady state with the elapse of time $\bar{t}$.

Figure 4 shows the variations of pulse EOF velocity with time for different relaxation times $\bar{\lambda}_{1}(0.01$ and 0.1$)$ when $K=20, a=1$ and $\bar{r}=0.5$. It is found that the time it takes for the fluid to change from a static state to a flowing state increases with relaxation time $\bar{\lambda}_{1}$. In this case, a possible explanation is that when the relaxation time is small, the viscoelasticity is not obvious. However, as the relaxation time $\bar{\lambda}_{1}$ increases, the viscoelasticity of the fluid becomes significant. In order to make the fluid flow, a part of the pulse force is needed to offset the resistance caused by viscoelasticity, so the time it takes for the fluid to change from a static state to a flowing state becomes longer. Meanwhile, it still can be found from Figure 4 that when the relaxation time is small (for example $\bar{\lambda}_{1}=0.01$ and $\left.\bar{\lambda}_{1}=0.1\right)$ and the time is in the range of 0 to $1(0 \leq \bar{t} \leq 1)$, the order of velocity amplitude from large to small is always rectangle pulse, triangle pulse, and sawtooth pulse. The reason is due to the influence of the nature of their different shaped pulses (see Figure 2). Furthermore, it is noted that no matter what kind of pulse, the velocity amplitude increases with relaxation time $\bar{\lambda}_{1}$. The reason for this fact is that the longer relaxation time means the larger elastic effect of the Maxwell fluid and results in larger velocity amplitude.

Figure 5 and Figure 6 depict the variations of pulse EOF velocity with time for different pulses (rectangle pulse, triangle pulse and sawtooth pulse) when $\bar{\lambda}_{1}=0.01$ and $\bar{\lambda}_{1}=0.1 \quad(K=20, \bar{r}=0.5)$, respectively. It still can be noted

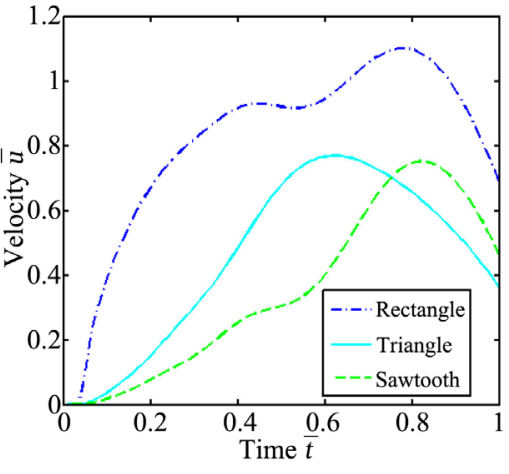

(a)

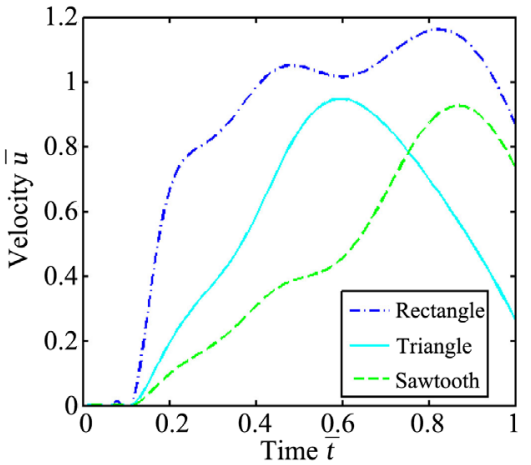

(b)

Figure 4. Variation of pulse EOF velocity $\bar{u}$ at different pulses with time for different relaxation times $\bar{\lambda}_{1}$ when $K=20, a=1$ and $\bar{r}=0.5$. (a) $\bar{\lambda}_{1}=0.01$; (b) $\bar{\lambda}_{1}=0.1$. 


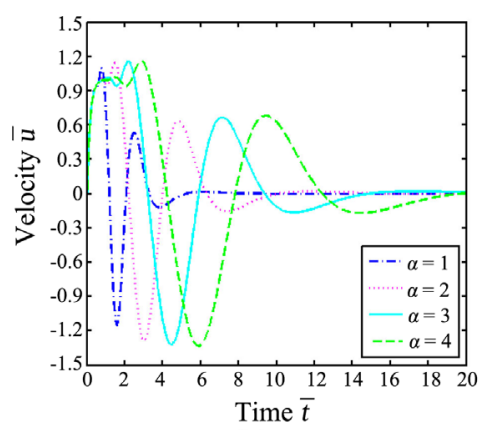

(a)

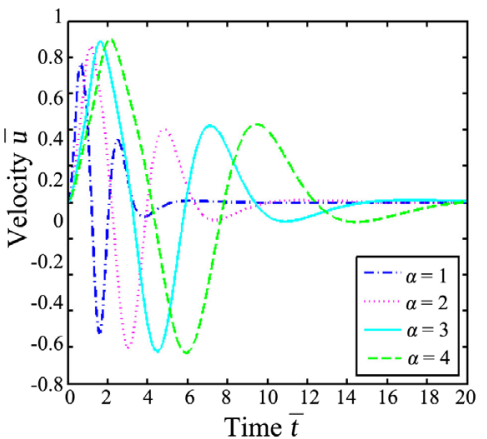

(b)

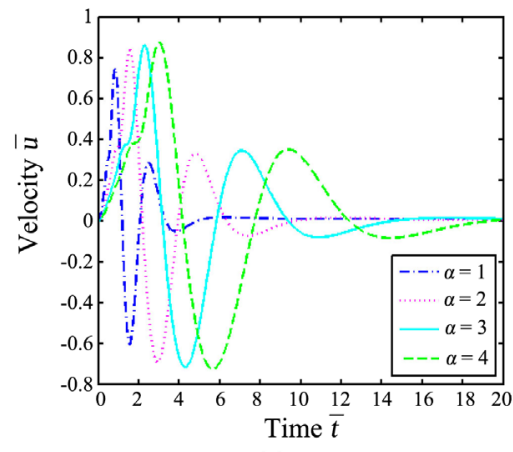

(c)

Figure 5. Variations of pulse EOF velocity $\bar{u}$ at different pulse widths with time for different pulses when $K=20, \bar{r}=0.5$ and $\bar{\lambda}_{1}=0.01$. (a) Rectangle pulse; (b) Triangle pulse; (c) Sawtooth pulse.

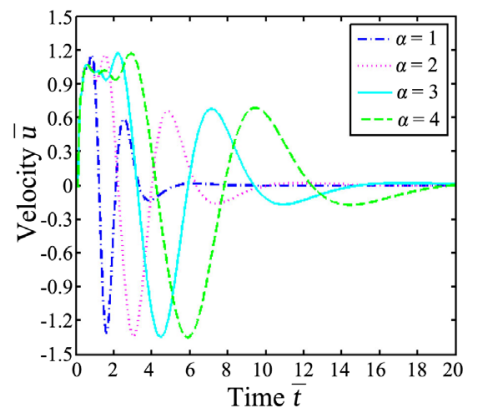

(a)

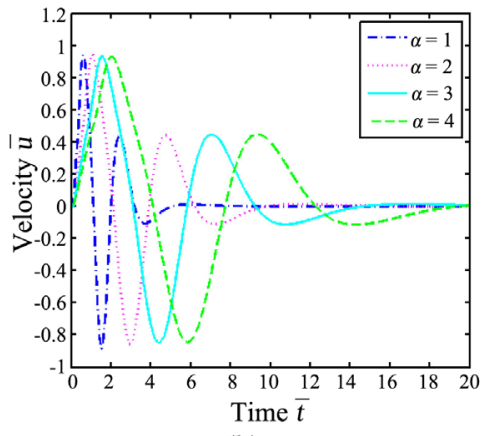

(b)

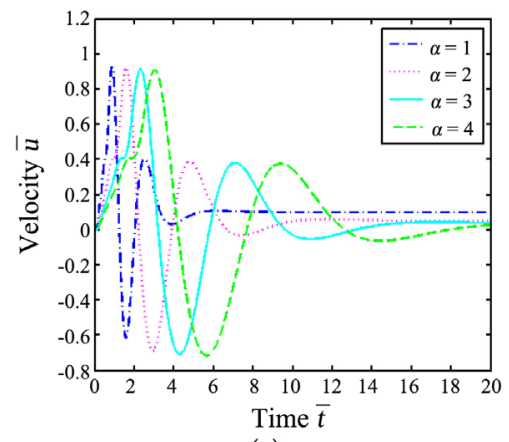

(c)

Figure 6. Variations of pulse EOF velocity $\bar{u}$ at different pulse widths with time for different pulses when $K=20, \bar{r}=0.5$ and $\bar{\lambda}_{1}=0.1$. (a) Rectangle pulse; (b) Triangle pulse; (c) Sawtooth pulse. 
that the amplitude of velocity increases with relaxation time $\bar{\lambda}_{1}$. At the same time, we can also be seen from both Figure 5 and Figure 6 that for different pulse widths $a$, the effect of relaxation time on the amplitude of different pulse EOF velocity is different. Especially for smaller pulse width is more significant (for example $a=1$ ). The reason may be that for small pulse width $a$, the pulse force has a short duration and the flow of fluid stability is relatively weak, so it is easily affected by other forces. In addition, we can see from the above picture that as the pulse width $a$ increases, the different change frequency of velocity profiles slows down, which means a long cycle time [37], and the time required for the velocity profiles to reach a steady state has also become longer.

Figure 7 and Figure 8 present the variations of pulse EOF velocity with radius for different pulses (rectangle pulse, triangle pulse and sawtooth pulse) when $\bar{\lambda}_{1}=0.1$ and $\bar{\lambda}_{1}=0.8 \quad(a=1, \bar{t}=0.5)$, respectively. It is clearly seen from Figure 7 that with the increase of electrokinetic width $K$ value, the pulse EOF velocity variations are mainly limited to the narrow area close to the EDL for small relaxation time (for example $\bar{\lambda}_{1}=0.1$ ). From Figure 8 , we can find that a larger relaxation time $\bar{\lambda}_{1}$ will result in larger velocity amplitude, and the velocity distribution is no longer mainly restricted to EDL. This is because that for a larger relaxation time $\bar{\lambda}_{1}$, the elasticity of the fluid is more obvious. And since elasticity is the physical property of the fluid as a whole, the velocity variation can extend to the entire region of the flow [38]. Additionally, by comparing the

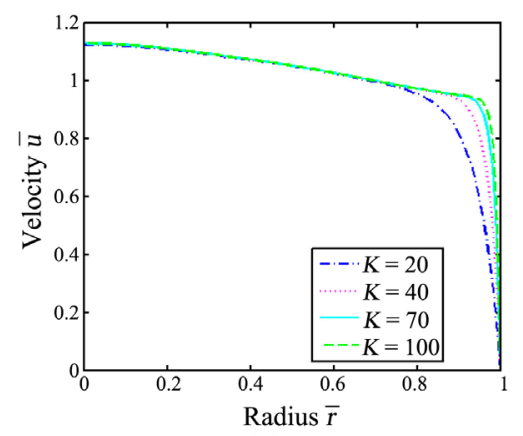

(a)

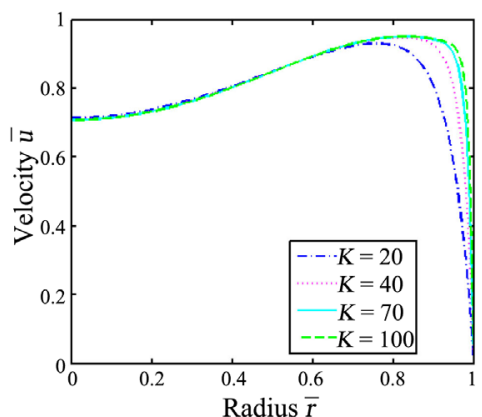

(b)

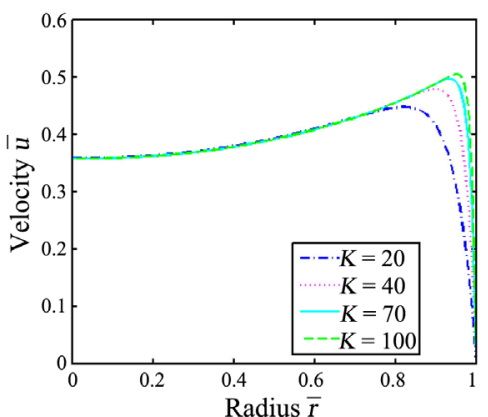

(c)

Figure 7. Variations of pulse EOF velocity $\bar{u}$ at different electrokinetic widths with radius for different pulses when $a=1, \bar{t}=0.5$ and $\bar{\lambda}_{1}=0.1$. (a) Rectangle pulse; (b) Triangle pulse; (c) Sawtooth pulse. 


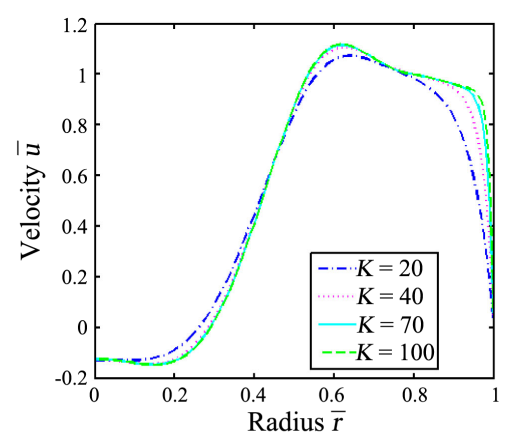

(a)

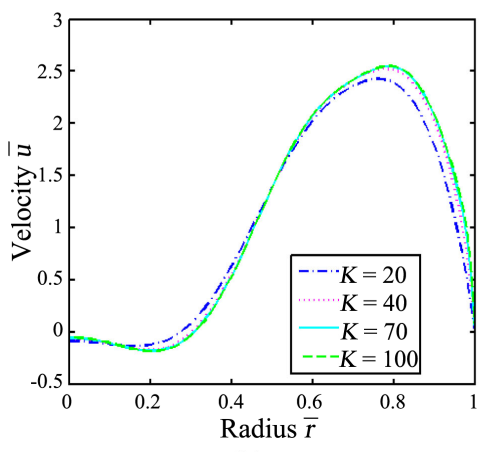

(b)

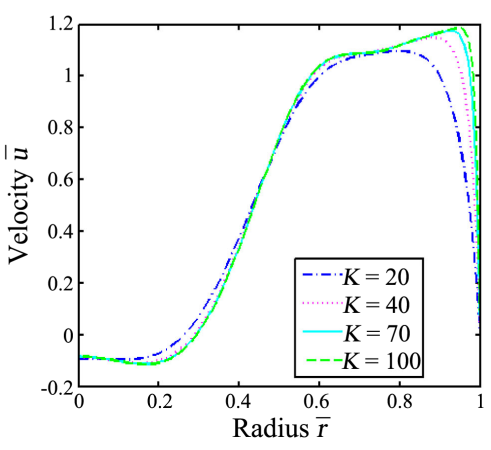

(c)

Figure 8. Variations of pulse EOF velocity $\bar{u}$ at different electrokinetic widths with radius for different pulses when $a=1, \bar{t}=0.5$ and $\bar{\lambda}_{1}=0.8$. (a) Rectangle pulse; (b) Triangle pulse; (c) Sawtooth pulse.

variations in the amplitude of the three kinds of pulse EOF velocity, we can see that the relaxation time has different effects on the velocity amplitude for different pulses. In particular, it has a greater impact on triangle pulse and sawtooth pulse than rectangle pulse (see Figure 7(b) and Figure 7(c), Figure 8(b) and Figure 8(c)). The possible reason is that the velocity expressions of triangle pulse EOF and sawtooth pulse EOF have one more variable about the relaxation time $\bar{\lambda}_{1}$ (see Equation (32) and Equation (35)) than that of rectangle pulse EOF. Thus, the rectangle pulse EOF is a relatively more stable pulse EOF among the three kinds of pulse EOF.

\section{Conclusion}

In this article, the semi-analytical solutions for both triangle and sawtooth time periodic pulse EOF of Maxwell fluid through a circular microchannel under the Debye-Hückel approximation are presented. Based on the results obtained in this work, it can be concluded that with the electrokinetic width $K$ increases, the velocity variations are mainly limited to the narrow area close to the EDL for

small relaxation time $\bar{\lambda}_{1}$. However, as the increase of relaxation time $\bar{\lambda}_{1}$, the elasticity of the fluid becomes significant and the velocity variations can be extended to the entire flow field. At the same time, the velocity amplitude will significantly larger, and the flow needs longer time to attain steady status. Moreover, the time it takes for the fluid to change from a static state to a flowing state 
increases with relaxation time $\bar{\lambda}_{1}$. For given pulse width $a$, the effect of relaxation time $\bar{\lambda}_{1}$ on triangle pulse EOF and sawtooth pulse EOF is greater than rectangle pulse EOF, which implies that the rectangle pulse EOF is more stable. With the increase of pulse width $a$, the effect of relaxation time $\bar{\lambda}_{1}$ on the velocity will be weakened, the change period of the velocity profiles becomes larger, and the time required for the velocity profiles to reach a steady state also becomes longer.

\section{Funding}

This work was supported by the Scientific Research Project of Inner Mongolia University of Technology (Grant No. ZZ201813).

\section{Conflicts of Interest}

The authors declare no conflicts of interest regarding the publication of this paper.

\section{References}

[1] Stone, H.A., Stroock, A.D. and Ajdari, A. (2004) Engineering Flows in Small Devices: Microfluidics toward a Lab-on-a-Chip. Annual Review of Fluid Mechanics, 36, 381. https://doi.org/10.1146/annurev.fluid.36.050802.122124

[2] Bayraktar, T. and Pidugu, S.B. (2006) Characterization of Liquid Flows in Microfluidic Systems. International Journal of Heat and Mass Transfer, 49, 815. https://doi.org/10.1016/j.ijheatmasstransfer.2005.11.007

[3] Hunter, R.J. (1981) Zeta Potential in Colloid Science. Academic Press, Cambridge.

[4] Levine, S., Marriott, J.R., Neale, G. and Epstein, N. (1975) Theory of Electrokinetic Flow in Fine Cylindrical Capillaries at High Zeta Potentials. Journal of Colloid \& Interface Science, 52, 136-149. https://doi.org/10.1016/0021-9797(75)90310-0

[5] Taos, H.K. (2000) Electroosmotic Flow through an Annulus. Journal of Colloid \& Interface Science, 225, 247-250. https://doi.org/10.1006/jcis.1999.6696

[6] Hsu, J.P., Kao, C.Y., Tseng, S.J. and Chen, C.J. (2002) Electrokinetic Flow through an Elliptical Microchannel: Effects of Aspect Ratio and Electrical Boundary Conditions. Journal of Colloid \& Interface Science, 248, 176-184. https://doi.org/10.1006/jcis.2001.8200

[7] Yang, C., Li, D. and Masliyah, J.H. (1998) Modeling Forced Liquid Convection in Rectangular Microchannels with Electrokinetic Effects. International Journal of Heat \& Mass Transfer, 41, 4229-4249. https://doi.org/10.1016/S0017-9310(98)00125-2

[8] Bianchi, F., Ferrigno, R. and Girault, H.H. (2000) Finite Element Simulation of an Electroosmotic Driven Flow Division at a t-Junction of Microscale Dimensions. Analytical Chemistry, 72, 1987-1993. https://doi.org/10.1021/ac991225z

[9] Wang, C.Y., Liu, Y.H. and Chang, C.C. (2008) Analytical Solution of Electro-Osmotic Flow in a Semicircular Microchannel. Physics of Fluids, 20, Article ID: 063105. https://doi.org/10.1063/1.2939399

[10] Dutta, P. and Beskok, A. (2001) Analytical Solution of Time Periodic Electroosmotic Flows: Analogies to Stokes' Second Problem. Analytical Chemistry, 73, 5097-5102. https://doi.org/10.1021/ac015546y 
[11] Kang, Y.J., Yang, C. and Huang, X.Y. (2002) Dynamic Aspects of Electroosmotic Flow in a Cylindrical Microcapillary. International Journal of Engineering Science, 40, 2203-2221. https://doi.org/10.1016/S0020-7225(02)00143-X

[12] Wang, X.M., Chen, B. and Wu, J.K. (2007) A Semianalytical Solution of Periodical Electro-Osmosis in a Rectangular Microchannel. Physics of Fluids, 19, Article ID: 127101. https://doi.org/10.1063/1.2784532

[13] Chakraborty, S. and Ray, S. (2008) Mass Flow-Rate Control through Time Periodic Electro-Osmotic Flows in Circular Microchannels. Physics of Fluids, 20, Article ID: 083602. https://doi.org/10.1063/1.2949306

[14] Jian, Y.J., Yang, L.G. and Liu, Q.S. (2010) Time Periodic Electro-Osmotic Flow through a Microannulus. Physics of Fluids, 22, Article ID: 042001.

https://doi.org/10.1063/1.3358473

[15] Deng, S.Y., Jian, Y.J., Bi, Y.H., Chang, L., Wang, H.J. and Liu, Q.S. (2010) Unsteady Electroosmotic Flow of Power-Law Fluid in a Rectangular Microchannel. Mechanics Research Communications, 39, 9-14.

https://doi.org/10.1016/j.mechrescom.2011.09.003

[16] Zhao, M.L. and Wang, S.W. (2015) Analytical Solution of the Transient Electro-Osmotic Flow of a Generalized Fractional Maxwell Fluid in a Straight Pipe with a Circular Cross-Section. European Journal of Mechanics B/ Fluids, 54, 82-86. https://doi.org/10.1016/j.euromechflu.2015.06.016

[17] Jian, Y.J., Su, J., Chang, L., Liu, Q.S. and He, G.W. (2014) Transient Electroosmotic Flow of General Maxwell Fluids through a Slit Microchannel. Zeitschrift fur angewandte Mathematik und Physik (ZAMP), 65, 435-447. https://doi.org/10.1007/s00033-013-0341-1

[18] Zhao, M.L., Wang, S.W. and Wei, S.S. (2013) Transient Electro-Osmotic Flow of Oldroyd-B Fluid in a Straight Pipe of Circular Cross Section. Journal of Non-Newtonian Fluid Mechanics, 201, 135-139. https://doi.org/10.1016/j.jnnfm.2013.09.002

[19] Jiang, Y.T., Qi, H.T., Xu, H.Y. and Jiang, X.Y. (2017) Transient Electroosmotic Slip Flow of Fractional Oldroyd-B Fluids. Microfluidics and Nanofluidics, 21, 1-10. https://doi.org/10.1007/s10404-016-1843-x

[20] Liang, P.C., Wang, S.W. and Zhao, M.L. (2020) Numerical Study of Rotating Electroosmotic Flow of Oldroyd-B Fluid in a Microchannel with Slip Boundary Condition. Chinese Journal of Physics, 65, 459-471. https://doi.org/10.1016/j.cjph.2020.02.025

[21] Xie, Z.Y. and Jian, Y.J. (2014) Rotating Electroosmotic Flow of Power-Law Fluids at High Zeta Potentials. Colloids \& Surfaces A: Physicochemical \& Engineering Aspects, 461, 231-239. https://doi.org/10.1016/j.colsurfa.2014.07.051

[22] Baos, R., Arcos, J., Bautista, O. and Mendez, F. (2018) Oscillatory Electroosmotic Flow of Power-Law Fluids in a Microchannel. International Journal of Mechanical \& Mechatronics, 12, 773-776.

[23] Baos, R., Arcos, J., Bautista, O. and Mendez, F. (2020) Slippage Effect on the Oscillatory Electroosmotic Flow of Power-Law Fluids in a Microchannel. Defect and Diffusion Forum, 399, 92-101. https://doi.org/10.4028/www.scientific.net/DDF.399.92

[24] Escandón, J., Jiménez, E., Hernández, C., Bautista, O. and Méndez, F. (2015) Transient Electroosmotic Flow of Maxwell Fluids in a Slit Microchannel with Asymmetric Zeta Potentials. European Journal of Mechanics - B/ Fluids, 53, 180-189. https://doi.org/10.1016/j.euromechflu.2015.05.001 
[25] Chu, X. and Jian, Y.J. (2019) Magnetohydrodynamic Electroosmotic Flow of Maxwell Fluids with Patterned Charged Surface in Narrow Confinements. Journal of Physics D Applied Physics, 52, Article ID: 405003. https://doi.org/10.1088/1361-6463/ab2b27

[26] Escandón, J., Torres, D., Hernández, C. and Vargas, R. (2020) Start-Up Electroosmotic Flow of Multi-Layer Immiscible Maxwell Fluids in a Slit Microchannel. Micromachines, 11, 757. https://doi.org/10.3390/mi11080757

[27] Shah, N.A., Mahsud, Y., Aziz, M. and Tlili, I. (2020) Analytical Solutions for Unsteady Electrohydrodynamics Flows of Maxwell Fluids in Microchannels with Circular Cross Section. Physics of Fluids, 32, Article ID: 013107. https://doi.org/10.1063/1.5128688

[28] Liu, Q.S., Jian, Y.J. and Yang, L.G. (2011) Alternating Current Electroosmotic Flow of the Jeffreys Fluids through a Slit Microchannel. Physics of Fluids, 23, 381. https://doi.org/10.1063/1.3640082

[29] Gao, C.H. and Jian, Y.J. (2015) Analytical Solution of Magnetohydrodynamic Flow of Jeffrey Fluid through a Circular Microchannel. Journal of Molecular Liquids, 211, 803-811. https://doi.org/10.1016/j.molliq.2015.08.004

[30] Li, F.Q., Jian, Y.J., Xie, Z.Y., Liu, Y.B. and Liu, Q.S. (2017) Transient Alternating Current Electroosmotic Flow of a Jeffrey Fluid through a Polyelectrolyte-Grafted Nanochannel. RSC Advances, 7, 782-790. https://doi.org/10.1039/C6RA24930B

[31] Fan, Y. and Cheng, Q. (2013) Analysis of Current on Human Therapeutic Effects in Low Frequency Pulse. Journal of Henan Institute of Science and Technology, 41, 45-47.

[32] Li, D.S. and Li, K. (2021) Analysis of Transient Pulse Electroosmotic Flow of Maxwell Fluid through a Circular Micro-Channel Using Laplace Transform Method. Open Journal of Fluid Dynamics, 11. (In Press)

[33] Na, R., Jian, Y.J., Chang, L., Su, J. and Liu, Q.S. (2013) Transient Electro-Osmotic and Pressure Driven Flows through a Microannulus. Open Journal of Fluid Dynamics, 3, 50-56. https://doi.org/10.4236/ojfd.2013.32007

[34] Bird, R.B., Stewart, W.E. and Lightfoot, E.N. (2002) Transport Phenomena, Second Edition. John Wiley \& Sons, Inc., New York.

[35] Hoog, F.D., Knight, J.H. and Stokes, A.N. (1982) An Improved Method for Numerical Inversion of Laplace Transforms. SIAM Journal on Scientific and Statistical, 3, 357-366. https://doi.org/10.1137/0903022

[36] Liu, Q.S., Yang, L.G. and Su, J. (2013) Transient Electroosmotic Flow of General Jeffrey Fluid between Two Micro-Parallel Plates. Acta Physica Sinica, 62, 1691-1702. https://doi.org/10.7498/aps.62.144702

[37] Yin, Z. (2014) Unsteady Electroosmotic Flow of Maxwell Fluids through a Parallel Plate Micro-Channel. Inner Mongolia University, Hohhot.

[38] Liu, Q.S., Jian, Y.J. and Yang, L.G. (2011) Time Periodic Electroosmotic Flow of the Generalized Maxwell Fluids between Two Micro-Parallel Plates. Journal of NonNewtonian Fluid Mechanics, 166, 478-486. https://doi.org/10.1016/j.jnnfm.2011.02.003 


\section{Appendix A. The Laplace Transform of Ideal Rectangle Pulse}

The Laplace transform of ideal rectangle pulse is expressed as follows [32]

$$
\begin{aligned}
\int_{0}^{\infty} f(\bar{t}) \mathrm{e}^{-s \bar{t}} \mathrm{~d} \bar{t} & =\int_{0}^{T} f(\bar{t}) \mathrm{e}^{-s \bar{t}} \mathrm{~d} \bar{t}+\int_{T}^{\infty} f(\bar{t}) \mathrm{e}^{-s \bar{t}} \mathrm{~d} \bar{t} \\
& =\int_{0}^{T} f(\bar{t}) \mathrm{e}^{-s \bar{t}} \mathrm{~d} \bar{t}+\mathrm{e}^{-s T} \int_{0}^{\infty} f(\tau) \mathrm{e}^{-s \tau} \mathrm{d} \tau \quad(\text { Let } \bar{t}=T+\tau)
\end{aligned}
$$

here $T=2 a$. It needs to point out that all of the following pulses have a period of $2 a$.

By shifting the term of Equation (A.1) and using the Equation (10), we can get

$$
\begin{aligned}
F(s) & =\int_{0}^{\infty} f(\bar{t}) \mathrm{e}^{-s \bar{t}} \mathrm{~d} \bar{t}=\frac{1}{1-\mathrm{e}^{-s T}} \int_{0}^{T} f(\bar{t}) \mathrm{e}^{-s \bar{t}} \mathrm{~d} \bar{t} \\
& =\frac{1}{1-\mathrm{e}^{-2 a s}} F_{1}(s)=\frac{1}{s} \tanh \left(\frac{a s}{2}\right)
\end{aligned}
$$

where

$$
\begin{aligned}
F_{1}(s) & =\int_{0}^{a} f(\bar{t}) \mathrm{e}^{-s \bar{t}} \mathrm{~d} \bar{t}+\int_{a}^{2 a} f(\bar{t}) \mathrm{e}^{-s \bar{t}} \mathrm{~d} \bar{t} \\
& =\frac{1-\mathrm{e}^{-a s}}{s}-\frac{\mathrm{e}^{-2 a s}\left(\mathrm{e}^{a s}-1\right)}{s}=\frac{\mathrm{e}^{-2 a s}\left(\mathrm{e}^{a s}-1\right)^{2}}{s}
\end{aligned}
$$

\section{Appendix B. The Laplace Transform of Ideal Triangle Pulse}

With the aid of Equation (A.1) and Equation (A.2), the Laplace transform of ideal triangle pulse can be given as

$$
\begin{aligned}
& G(s)=\int_{0}^{\infty}\left(1+\bar{\lambda}_{1} \frac{\partial}{\partial t}\right) f(\bar{t}) \mathrm{e}^{-s \bar{t}} \mathrm{~d} \bar{t}=\frac{1}{1-\mathrm{e}^{-s T}} \int_{0}^{T}\left(1+\bar{\lambda}_{1} \frac{\partial}{\partial t}\right) f(\bar{t}) \mathrm{e}^{-s \bar{t}} \mathrm{~d} \bar{t} \\
& =\frac{1}{1-\mathrm{e}^{-s T}}\left(G_{1}(s)+\bar{\lambda}_{1} G_{2}(s)\right) \\
& =\frac{1}{1-\mathrm{e}^{-2 a s}}\left\{\frac{2 \mathrm{e}^{-2 a s}\left(-1+\mathrm{e}^{\frac{a s}{2}}\right)^{3}\left(1+\mathrm{e}^{\frac{a s}{2}}\right)}{a s^{2}}+\bar{\lambda}_{1} \frac{\mathrm{e}^{-a s}\left(-8 \sinh \left(\frac{a s}{2}\right)+4 \sinh (a s)\right)}{a s\left(1-\mathrm{e}^{-2 a s}\right)}\right\} \\
& =\frac{2-2 \operatorname{sech}\left(\frac{a s}{2}\right)}{a s^{2}}+\bar{\lambda}_{1} \frac{2-2 \operatorname{sech}\left(\frac{a s}{2}\right)}{a s}=\frac{2\left(1+\bar{\lambda}_{1} s\right)\left(1-\operatorname{sech}\left(\frac{a s}{2}\right)\right)}{a s^{2}}
\end{aligned}
$$

where

$$
\begin{aligned}
& G_{1}(s)=\int_{0}^{\frac{a}{2}} f(\bar{t}) \mathrm{e}^{-s \bar{t}} \mathrm{~d} \bar{t}+\int_{\frac{a}{2}}^{\frac{3 a}{2}} f(\bar{t}) \mathrm{e}^{-s \bar{t}} \mathrm{~d} \bar{t}+\int_{\frac{3 a}{2}}^{2 a} f(\bar{t}) \mathrm{e}^{-s \bar{t}} \mathrm{~d} \bar{t} \\
& =\frac{2\left(1-\frac{1}{2} \mathrm{e}^{-\frac{a s}{2}}(2+a s)\right)}{a s^{2}}+\frac{\mathrm{e}^{-\frac{3 a s}{2}}\left(2+a s+\mathrm{e}^{a s}(-2+a s)\right)}{a s^{2}}+\frac{\mathrm{e}^{-2 a s}\left(-2+\mathrm{e}^{\frac{a s}{2}}(2-a s)\right)}{a s^{2}} \\
& =\frac{2 \mathrm{e}^{-2 a s}\left(-1+\mathrm{e}^{\frac{a s}{2}}\right)^{3}\left(1+\mathrm{e}^{\frac{a s}{2}}\right)}{a s^{2}}
\end{aligned}
$$




$$
\begin{aligned}
G_{2}(s) & =\int_{0}^{\frac{a}{2}} \frac{\partial}{\partial t} f(\bar{t}) \mathrm{e}^{-s \bar{t}} \mathrm{~d} \bar{t}+\int_{\frac{a}{2}}^{\frac{3 a}{2}} \frac{\partial}{\partial t} f(\bar{t}) \mathrm{e}^{-s \bar{t}} \mathrm{~d} \bar{t}+\int_{\frac{3 a}{2}}^{2 a} \frac{\partial}{\partial t} f(\bar{t}) \mathrm{e}^{-s \bar{t}} \mathrm{~d} \bar{t} \\
& =\frac{2-2 \mathrm{e}^{-\frac{a s}{2}}}{a s^{2}}-\frac{2 \mathrm{e}^{-\frac{3 a s}{2}}\left(-1+\mathrm{e}^{a s}\right)}{a s^{2}}+\frac{2 \mathrm{e}^{-2 a s}\left(-1+\mathrm{e}^{\frac{a s}{2}}\right)}{a s^{2}} \\
& =\frac{\mathrm{e}^{-a s}\left(-8 \sinh \left(\frac{a s}{2}\right)+4 \sinh (a s)\right)}{a s\left(1-\mathrm{e}^{-2 a s}\right)}
\end{aligned}
$$

\section{Appendix C. The Laplace Transform of Ideal Sawtooth Pulse}

Similarly, the Laplace transform of ideal sawtooth pulse with Equation (A.1) and Equation (A.2) can be written as

$$
\begin{aligned}
H(s) & =\int_{0}^{\infty}\left(1+\bar{\lambda}_{1} \frac{\partial}{\partial t}\right) f(\bar{t}) \mathrm{e}^{-s \bar{t}} \mathrm{~d} \bar{t}=\frac{1}{1-\mathrm{e}^{-s T}} \int_{0}^{T}\left(1+\bar{\lambda}_{1} \frac{\partial}{\partial t}\right) f(\bar{t}) \mathrm{e}^{-s \bar{t}} \mathrm{~d} \bar{t} \\
& =\frac{1}{1-\mathrm{e}^{-s T}}\left(H_{1}(s)+\bar{\lambda}_{1} H_{2}(s)\right) \\
& =\frac{1}{1-\mathrm{e}^{-2 a s}}\left(\frac{1-\mathrm{e}^{-2 a s}-2 a s \mathrm{e}^{-a s}}{a s^{2}}+\bar{\lambda}_{1} \frac{1-\mathrm{e}^{-2 a s}}{a s}\right) \\
& =\frac{1-a s \operatorname{csch}(a s)}{a s^{2}}+\bar{\lambda}_{1} \frac{1}{a s}=\frac{1+\bar{\lambda}_{1} s-a s \operatorname{csch}(a s)}{a s^{2}}
\end{aligned}
$$

where

$$
\begin{aligned}
H_{1}(s) & =\int_{0}^{a} f(\bar{t}) \mathrm{e}^{-s \bar{t}} \mathrm{~d} \bar{t}+\int_{a}^{2 a} f(\bar{t}) \mathrm{e}^{-s \bar{t}} \mathrm{~d} \bar{t} \\
& =\frac{1-\mathrm{e}^{-a s}(1+a s)}{a s^{2}}+\frac{\mathrm{e}^{-2 a s}\left(-1+\mathrm{e}^{a s}(1-a s)\right)}{a s^{2}} \\
& =\frac{1-\mathrm{e}^{-2 a s}-2 a s \mathrm{e}^{-a s}}{a s^{2}} \\
H_{2}(s) & =\int_{0}^{a} \frac{\partial}{\partial t} f(\bar{t}) \mathrm{e}^{-s \bar{t}} \mathrm{~d} \bar{t}+\int_{a}^{2 a} \frac{\partial}{\partial t} f(\bar{t}) \mathrm{e}^{-s \bar{t}} \mathrm{~d} \bar{t} \\
& =\frac{1-\mathrm{e}^{-\frac{a s}{2}}}{a s^{2}}+\frac{\mathrm{e}^{-2 a s}\left(-1+\mathrm{e}^{a s}\right)}{a s} \\
& =\frac{1-\mathrm{e}^{-2 a s}}{a s}
\end{aligned}
$$

